\title{
La création de la Cour africaine des droits de l'Homme et des peuples. Les dessous d'une ingénierie institutionnelle multicentrée
}

\section{Marielle Debos}

\section{(2) OpenEdition \\ Journals}

Édition électronique

URL : https://journals.openedition.org/conflits/1934

DOI : $10.4000 /$ conflits. 1934

ISSN : 1777-5345

Éditeur :

CECLS - Centre d'études sur les conflits - Liberté et sécurité, L'Harmattan

Édition imprimée

Date de publication : 1 décembre 2005

Pagination : 159-182

ISBN : 2-296-00230-7

ISSN : 1157-996X

Référence électronique

Marielle Debos, «La création de la Cour africaine des droits de l'Homme et des peuples. Les dessous d'une ingénierie institutionnelle multicentrée », Cultures \& Conflits [En ligne], 60 | hiver 2005, mis en ligne le 23 février 2006, consulté le 27 septembre 2021. URL : http://journals.openedition.org/conflits/ 1934 ; DOI : https://doi.org/10.4000/conflits. 1934

Ce document a été généré automatiquement le 27 septembre 2021

Creative Commons License 


\title{
La création de la Cour africaine des droits de l'Homme et des peuples. Les dessous d'une ingénierie institutionnelle multicentrée
}

\author{
Marielle Debos
}

1 Les acteurs non étatiques ont traditionnellement joué un rôle important dans l'élaboration et la mise en œuvre des normes et des codes moraux de la scène internationale ${ }^{1}$. Aujourd'hui les droits humains ont le vent en poupe. Portées par les Etats les plus puissants et par une multitude d'acteurs non étatiques, ces nouvelles normes ont envahi la scène internationale. Malgré la persistance des violations des droits de l'Homme, aucun acteur - étatique ou non - ne peut aujourd'hui revendiquer la transgression de cette norme. Celle-ci a en effet été adoptée, à des degrés divers et selon des modalités différentes, par toutes les régions du monde. Ce ne sont cependant pas uniquement les références et les discours qui se propagent, mais aussi les techniques de protection des droits humains qui s'exportent et s'importent. Les experts juridiques parcourent les continents et participent à la création de nouvelles règles et institutions. On a ainsi vu des "pèlerins constitutionnels » partir après la chute du rideau de fer vers les pays de l'est de l'Europe ${ }^{2}$, des réseaux transnationaux de militants ${ }^{3}$ contribuer à la diffusion de règles de droit condamnant les violences faites aux femmes et des organisations non gouvernementales (ONG) initier et négocier le statut de Rome de la Cour Pénale Internationale.

2 La création de la Cour africaine des droits de l'Homme et des peuples s'inscrit dans ce contexte de multiplication des lieux de production du droit et de diffusion des techniques de protection des droits humains. S'inspirant des deux précédents régionaux - la Cour européenne des droits de l'Homme et la Cour interaméricaine des droits de l'Homme, elle est censée pallier les insuffisances du mécanisme africain de protection des droits humains. Ce mécanisme, qui prend sa source dans la Charte africaine des droits de l'Homme et des peuples de 1981, repose sur une Commission 
dotée de peu de moyens qui cristallise les mécontentements et les déceptions. La Cour africaine des droits de l'Homme et des peuples, qui viendra bientôt compléter le travail de la Commission, est le fruit d'un long processus : après des années de négociation, le Protocole additionnel à la Charte portant création de la Cour est enfin adopté en juin 1998 à Ouagadougou au Burkina Faso. Il faut cependant attendre le 25 janvier 2004 pour qu'il entre en vigueur, trente jours après avoir été ratifié par quinze Etats membres de l'Union africaine $e^{4}$. Après deux ans d'existence virtuelle, la Cour pourrait voir le jour en 2006 si la Conférence des chefs d'Etat et de gouvernement de l'Union africaine désigne son siège, procède à l'élection des juges et lui alloue un budget permettant d'assurer son fonctionnement.

3 Le texte fondateur de la Cour, "le Protocole de Ouagadougou», présente les Etats africains et l'Organisation de l'unité africaine (OUA) - aujourd'hui l'Union africaine $(\mathrm{UA})^{5}$ - comme les seuls protagonistes de l'histoire de sa création. Mais une étude attentive de l'ingénierie institutionnelle révèle au contraire que les acteurs non étatiques ont joué un rôle central dans le processus : ils ont donné l'impulsion initiale, orchestré la mobilisation, encadré les négociations et milité pour l'adoption et la ratification du Protocole. Sans l'implication des organisations non gouvernementales, le projet de création de cette juridiction n'aurait certainement jamais été à l'agenda de l'organisation politique africaine.

4 Au-delà de la reconstitution de la genèse de la Cour africaine, l'enjeu de cette étude est de mettre en évidence les logiques de l'ingénierie institutionnelle et de donner vie à la problématique de la dynamique globale des droits humains en repérant les acteurs du changement et en dévoilant leurs stratégies. Il s'agit de dépasser la perspective diffusionniste qui surestime le poids des dynamiques et néglige le rôle de l'agent, individu ou institution, qui se lance dans une entreprise de production et de diffusion de normes. Les ONG internationales, et singulièrement leurs experts juridiques, participent de façon croissante à la production normative dans le domaine des droits humains. La prolifération des experts non gouvernementaux est cependant trop souvent saluée comme une évolution nécessairement prometteuse. Ce nouveau mode de production du droit doit au contraire être analysé avec un regard critique afin de mettre en évidence les luttes de pouvoir très fortes au sein du " monde multicentré ${ }^{6}$ (le monde des acteurs non étatiques). Quelles sont les ressources clés des entrepreneurs de normes : la mobilisation des militants et des individus, leurs capacités d'expertise ou leurs liens avec les notables des diplomaties d'Etat?

5 L'étude de l'ingénierie institutionnelle de cette juridiction panafricaine est révélatrice des nouvelles configurations du champ des droits humains. Il apparait tout d'abord qu'un petit nombre d'ONG internationales professionnalisées et bureaucratisées, spécialisées dans la production de standards juridiques, s'est imposé sur la scène internationale. Quelles sont les conséquences de cette institutionnalisation des acteurs non étatiques engagés dans la production de normes? Ce phénomène semble déboucher sur un partage des tâches entre les spécialistes et les militants des organisations «de terrain » qui n'ont ni les capacités d'expertise ni la maîtrise des codes sociaux nécessaires à la participation aux conférences internationales où les textes juridiques sont négociés en comités restreints. En Afrique, le fossé qui se creuse entre les militants et les professionnels est d'autant plus important que les ONG de protection des droits humains n'ont amorcé leur professionnalisation que dans les années 1990. Les inégalités entre les acteurs sociaux censés participer à la «société 
civile mondiale" sont ainsi particulièrement importantes dans le domaine très technique de la production normative.

6 Cette étude vise ensuite à mettre en évidence les multiples interactions entre le monde des Etats et le monde «multicentré ». Les liens entre les acteurs des deux mondes se resserrent: leurs besoins créent des relations d'interdépendance et ils sont souvent bien plus proches que ne le laissent apparaître leurs discours. En quoi la symbiose croissante des activités des diplomaties traditionnelles et des diplomaties privées affecte-t-elle l'identité des acteurs de la scène internationale? Il ne suffit cependant pas d'étudier les complémentarités entre les ONG et les Etats pour comprendre l'extraordinaire complexité de leurs relations et prendre la mesure de la porosité de la frontière qui les sépare. Il convient de mettre en évidence la proximité sociale des élites des milieux des ONG d'une part, et des milieux gouvernementaux et diplomatiques d'autre part. Les réseaux de sociabilité sont ainsi un atout de ces nouveaux notables du droit qui font figure de véritables $"$ paradiplomates $»^{7}$. Il s'agit finalement de savoir quels acteurs ont les moyens (capacités d'expertise, maîtrise des technologies juridiques importées, appartenance à des réseaux de notables) d'entrer dans le club fermé des producteurs et diffuseurs de standards juridiques.

7 Enfin, nous tenterons de comprendre les politiques adoptées par les Etats africains et par l'OUA/UA face à une initiative prise par des acteurs non gouvernementaux en vue de renforcer un mécanisme de protection des droits humains. Pourquoi les acteurs gouvernementaux africains ont-ils accepté l'option juridictionnelle, alors que, vingt ans auparavant, ils affirmaient leur attachement à ce qu'ils présentaient comme la tradition africaine de la conciliation? Qu'est-ce qui a changé depuis les années 1980 au cours desquelles les Etats avaient conçu le mécanisme de protection des droits de l'Homme comme "un simple habit de gala pour mondanités internationales $»^{8}$ ? Pour apporter des éléments de réponse à ces questions, il faut étudier le poids des normes dans un contexte de hausse du " coût de la dictature $»^{9}$ sur la scène internationale. Loin de considérer la création de la Cour africaine comme une "évolution " naturelle et inévitable, nous soulignerons le rôle des individus et des groupes engagés dans la promotion des droits humains, ainsi que l'influence du contexte global et régional.

La production de normes : une affaire de spécialistes

8 Les institutions sont trop souvent considérées comme un donné ou comme la réponse à une impérieuse nécessité de modernisation. Si les contraintes et les opportunités offertes par le contexte international ne sauraient être négligées, la création d'institutions de protection des droits humains en Afrique ne peut être réduite à un processus inévitable d'adaptation aux normes dominantes ou à un phénomène naturel d'attraction pour les technologies inventées sur d'autres continents et érigées en modèles. C'est en effet souvent moins le besoin d'innovations que la mobilisation des entrepreneurs de normes qui lance le processus de l'ingénierie institutionnelle. Pour Yves Mény, il existe «un marché institutionnel dans lequel, entre des offreurs (...) et les demandeurs (...), s'insèrent des entrepreneurs (...) qui servent de médiateurs et surtout d'interprètes des solutions qui leur paraissent désirables $»^{10}$. Dans le cas du mécanisme africain de protection des droits de l'Homme, le rôle de promoteurs des innovations revient à un groupe d'experts africains coordonné par une ONG internationale basée à Genève : la Commission Internationale des Juristes (CIJ).

Un processus d'ingénierie institutionnelle orchestré par la Commission Internationale des Juristes 
9 L'idée d'avoir recours au droit et aux institutions pour promouvoir et protéger les droits humains en Afrique apparaît pour la première fois en 1961 lors du Congrès des juristes africains organisé par la CIJ à Lagos au Nigeria. Ce Congrès sur « la primauté du droit » interpelle les dirigeants des puissances coloniales et des Etats africains indépendants afin qu'ils étudient la possibilité d'élaborer « une Convention africaine des droits de l'Homme prévoyant notamment la création d'un tribunal approprié et des voies de recours ouvertes à toutes les personnes relevant de la juridiction des Etats signataires $»^{11}$. « La loi de Lagos ", reprise par plusieurs congrès de juristes africains, devient une référence.

10 Au cours des années 1970, la CIJ mobilise les élites du continent autour du projet d'une Charte africaine des droits de l'Homme. Celle-ci est finalement élaborée en 1979 par un groupe d'experts constitué sous la présidence de Kéba Mbaye qui est alors à la tête de la CIJ. Le projet des experts non gouvernementaux est ensuite négocié par les représentants des Etats. La proposition de créer une Cour pour juger les crimes contre l'humanité et assurer la protection des droits de l'Homme est longuement discutée avant d'être rejetée par les Etats réticents à toute ingérence dans leurs affaires intérieures et soucieux de préserver une indépendance fraîchement acquise. La raison officielle de ce refus est cependant que le système de règlement judiciaire des différends ne correspond pas à la «tradition africaine de la conciliation et de l'arbre à palabres ». La "Charte de Banjul » est finalement adoptée en 1981 par l'Assemblée des chefs d'Etat et de gouvernement de l'OUA sans débat ni vote formel ${ }^{12}$. Elle entre en vigueur le 21 octobre 1986 et donne naissance à la Commission africaine des droits de l'Homme et des peuples ${ }^{13}$ qui a pour mandat la promotion et la protection des droits humains ${ }^{14}$.

11 La création de la Cour africaine est entreprise, au début des années 1990, par une nouvelle génération de responsables de la $\mathrm{CIJ}$ menée par Adama Dieng, un juriste sénégalais qui a été formé et introduit dans les milieux gouvernementaux et non gouvernementaux par son prédécesseur, Kéba Mbaye. L'élaboration du Protocole de Ouagadougou se déroule selon le même schéma que l'élaboration de la Charte africaine : les nouveaux dirigeants de la CIJ reprennent les stratégies de leurs aînés pour mobiliser les élites, élaborer un projet, orchestrer les négociations officielles et obtenir l'adoption et la ratification du texte.

Les conditions des innovations : engagement des entrepreneurs de normes et effets de contexte

12 Pourquoi le projet de la création d'une juridiction a-t-il été repris au début des années 1990 alors qu'il avait échoué quinze ans auparavant? La première condition de l'engagement des élites dans un programme d'innovation institutionnelle est l'insatisfaction à l'égard du système en vigueur ${ }^{15}$. Au début de la décennie 1990, les défenseurs des droits humains engagés sur le continent s'accordent pour dénoncer l'inefficacité de la Commission africaine des droits de l'Homme et des peuples. La Charte africaine ne donne tout d'abord pas le pouvoir de décision à la Commission ${ }^{16}$ : elle peut enquêter, tenter un arrangement amiable, mais elle doit adresser son rapport pour décision et éventuellement publication à l'Assemblée des chefs d'Etat et de gouvernement de l'OUA ${ }^{17}$ (aujourd'hui la Conférence des chefs d'Etat et de gouvernement de l'UA). Les décisions sont en général prises par consensus, de telle sorte que chaque membre possède de facto un droit de veto. La Charte n'a donc pas donné à la Commission les moyens d'intervenir efficacement en cas de violations des 
droits humains. Les échecs s'accumulant ${ }^{18}$, les entrepreneurs de normes vont recourir à des solutions pratiquées ailleurs pour pallier les faiblesses du système.

La seconde condition du changement institutionnel est un contexte favorable. Au moment de l'élaboration de la Charte de Banjul, l'idée de la création d'une Cour africaine n'avait pas été retenue pour des raisons essentiellement politiques. Mais, au début des années 1990, le vent tourne avec la fin de la Guerre Froide. Au sein de l'OUA les normes - attentes collectives sur le comportement correct pour une identité donnée ${ }^{19}$ - changent : on parle moins de la défense de la souveraineté et plus des droits humains ${ }^{20}$. Ce nouveau discours est utilisé comme un levier par les ONG qui dénoncent le gouffre entre les discours et les pratiques. Le projet de la création d'une Cour africaine des droits de l'Homme semble d'autant plus réalisable que l'on assiste depuis les années 1980 à un reflux de la méfiance des pays du continent pour le règlement judiciaire des différends ${ }^{21}$ : les Etats africains saisissent la Cour Internationale de Justice et participent aux négociations qui ont donné corps à la Cour Pénale Internationale ${ }^{22}$. Enfin, ce n'est pas seulement le contexte global qui change mais aussi le regard des acteurs sur les enjeux internationaux. Selon un haut fonctionnaire du Haut Commissariat des droits de l'Homme de l'ONU :

«Ce n'est pas un hasard si l'idée de créer la Cour a été reprise en 1993. C'était l'époque où les droits de l'Homme commençaient à être mieux reconnus. C'était juste après la Conférence de Vienne ${ }^{23}$. Le moment était vraiment favorable... plus que maintenant $»^{24}$.

14 Ainsi, au début des années 1990, les acteurs engagés sur le continent s'accordent sur la nécessité d'améliorer le mécanisme existant, tandis que le climat politique offre de nouvelles fenêtres d'opportunités. Il ne manque qu'un petit groupe d'entrepreneurs de normes susceptibles de se lancer dans l'aventure.

Mobilisation des notables et consultation des militants

Toute entreprise de production de normes commence avec le rassemblement des élites et des militants autour d'un projet. La mobilisation pour la Cour africaine n'a pas été l'œuvre d'une coalition d'ONG - comme ce fut le cas pour la Convention d'Ottawa sur les mines antipersonnel ou la Cour Pénale Internationale -, mais d'une ONG internationale caractéristique de ces nouveaux notables du droit spécialistes de la diffusion des modèles juridiques ${ }^{25}$ : la Commission Internationale des Juristes. Le rôle de la CIJ à cette étape de l'impulsion du processus est crucial. Selon un acteur impliqué dans la création de la Cour :

« La CIJ avait de l'influence en Afrique. Elle avait le leadership pour organiser la coopération des ONG avec la Commission africaine. Cela lui a permis de développer l'idée que la Commission était inefficace et qu'il fallait créer une Cour pour y remédier $»^{26}$.

C'est en janvier 1993, lors des « Journées de réflexion de haut niveau », que les notables africains réunis par la $\mathrm{CIJ}$ décident de lancer le processus de la création de la Cour. La CIJ consulte ensuite les membres des ONG africaines « de terrain »: en novembre 1993, elle présente son projet au forum des ONG qui précède chacune des deux sessions annuelles de la Commission africaine des droits de l'Homme ${ }^{27}$. Il s'agit d'une proposition de Protocole additionnel à la Charte africaine rédigée par Karel Vasak, un juriste tchèque reconnu dans le champ des droits humains. Le projet est cependant vivement critiqué par les militants qui le considèrent comme une copie irréfléchie du mécanisme européen. Les représentants des ONG africaines s'opposent en particulier à la suppression de la Commission et à son remplacement par la Cour; ils acceptent 
l'option juridictionnelle à condition de pouvoir continuer à travailler avec la Commission. Les participants au forum estiment en effet que les organes juridictionnels, qui ne permettent pas la participation des non-juristes, ont une approche très « légaliste » et très étroite des droits humains. Après ce faux pas, la CIJ revoit sa stratégie et recrute cinq experts «non gouvernementaux $»^{28}$ africains pour rédiger un nouveau projet. Après cette première étape de la consultation des militants, l'ingénierie institutionnelle passe entre les mains des professionnels de la production normative ; les ONG locales doivent désormais se contenter d'un rôle de figurant. Si certaines ONG africaines se sont professionnalisées depuis le début des années 1990, elles ne peuvent rivaliser avec les grandes ONG internationales spécialistes du droit.

Les logiques de la marginalisation : un partage des tâches entre les experts et les militants

L'ingénierie institutionnelle est toujours une affaire de spécialistes. La technicisation de la protection des droits humains et l'autonomisation de ce champ, qui est son corollaire, donnent en effet un essor considérable à l'expertise. La diffusion du modèle juridictionnel vers l'Afrique est ainsi l'œuvre d'une communauté épistémique réunissant des experts africains qui maitrisent ces technologies juridiques. Ceux-ci sont issus d'une nouvelle classe de professionnels africains des droits humains formés dans les universités européennes et nord-américaines. Depuis le début des années 1990, ils occupent des postes à responsabilité au sein des grandes ONG internationales, à l'instar d'Adama Dieng, secrétaire de la CIJ au moment de l'élaboration du Protocole de Ouagadougou.

Dans ce contexte de forte professionnalisation du champ des droits humains, la marginalisation des militants locaux relève non pas d'un complot des initiés, bâtisseurs patentés d'institutions, mais de l'impossibilité pour les non-spécialistes de participer activement à un processus qui exige de grandes capacités d'expertise. La maîtrise des instruments juridiques mais aussi des codes sociaux des milieux à conquérir sont des éléments très discriminants. En Afrique comme ailleurs, on observe ainsi un partage des tâches entre les professionnels et «les autres » : aux professionnels la production normative, aux militants le travail de terrain.

19 Ce phénomène n'est pas anodin : les ONG les plus puissantes ont tendance à se poser en représentantes de l'ensemble des défenseurs des droits humains. Tout se passe comme si les professionnels du droit étaient les porte-parole des militants locaux. Pourtant, ces représentants auto-institués ont des préoccupations bien différentes de celles des militants pour qui ils affirment travailler : la création d'une juridiction n'a jamais été une priorité des acteurs locaux. Le recours au mécanisme africain de protection des droits humains apparait d'ailleurs assez éloigné de la culture militante des acteurs « de terrain » qui préfèrent souvent mener des actions au niveau national ou local. Pourquoi croiraient-ils en un mécanisme importé d'un répertoire qui ne leur est pas familier? Pourquoi investiraient-ils dans un processus aussi hasardeux quand des professionnels s'en chargent ? Un responsable d'une ONG togolaise explique :

«Aujourd'hui personne [au sein des ONG africaines de terrain] ne s'intéresse à cette Cour. Ce n'est pas notre priorité. Nous ne pouvons risquer de perdre notre temps à travailler avec des organes de l'OUA. On milite plutôt pour des solutions immédiates. On travaille dans l'urgence. (...) Les ONG internationales ont suffisamment de moyens et font un travail important. Elles s'intéressent à ces grands projets à long terme. On n'a pas les moyens de développer une activité de suivi efficace. (...) C'est la même chose avec la Commission africaine. On ne perd pas 
notre temps car on sait que la Commission est inefficace et qu'elle n'a aucun pouvoir sur les Etats. On laisse ce travail aux experts $»^{29}$. peut être entreprise que par des experts qui entretiennent des relations de collaboration, et non d'affrontement direct et systématique, avec les Etats et les organisations intergouvernementales. L'enjeu pour la CIJ et les autres ONG qui souhaitent participer à l'écriture du droit est d'être jugées dignes de confiance par leurs interlocuteurs étatiques et intergouvernementaux. Ainsi la CIJ tient-elle beaucoup à son statut d'organisation réunissant des experts apolitiques. Selon Howard Tolley :

« La CIJ n'est pas et n'a jamais été un mouvement spontané de citoyens organisé pour défier le pouvoir d'Etat. (...) [Elle] ressemble plus aux organisations quasi-non gouvernementales qui utilisent des fonds publics pour fournir un service public $»^{30}$. institutionnalisation. La spécialisation dans la production de normes les éloigne des militants de terrain et les rapproche des cercles du pouvoir, l'efficacité de leur action dépendant moins du nombre de leurs adhérents que du prestige social et de l'intelligence politique de leurs leaders. Ces ONG spécialistes du droit international sont alors moins éloignées des diplomaties d'Etat que ne le laissent apparaître leurs discours.

Des interdépendances institutionnalisées aux relais informels des réseaux de notables

Si les experts « non gouvernementaux » sont à l'origine de la gestation du Protocole de Ouagadougou, les Etats ne sont pas pour autant évincés du processus d'élaboration du droit. Le texte est négocié avec les Etats, destinataires ultimes de la norme produite. Pour comprendre les nouvelles modalités de la production normative sur la scène africaine comme sur la scène internationale, il faut être particulièrement attentif à l'évolution des relations des acteurs étatiques et non étatiques.

23

Selon James Rosenau, les habitants des deux mondes séparés, le « monde des Etats » et le « monde multicentré ", interagissent de manière à rendre leur coexistence possible ${ }^{31}$. Mais, au-delà de cette séparation, leurs liens se resserrent, les Etats étant de moins en moins réticents à l'action des ONG. Ce sont tout d'abord des logiques d'interdépendance qui lient les Etats, les organisations intergouvernementales et les acteurs non étatiques. Ensuite, avec la mobilité des élites qui passent facilement d'un monde à l'autre se développent des réseaux de notables qui contribuent à leur tour au développement des liens entre les différentes institutions. Ces deux niveaux d'analyse sont complémentaires et inséparables. L'observateur qui n'analyserait que les relations entre les Etats et les ONG ne comprendrait pas comment la $\mathrm{CIJ}$ a réussi à placer la création de la Cour sur l'agenda de l'OUA. Inversement, celui qui ne s'intéresserait qu'aux réseaux de notables passerait sous silence les enjeux de la redéfinition des identités des acteurs étatiques et non étatiques. C'est pourquoi nous analyserons successivement les logiques d'interdépendance et le développement des réseaux de notables en mettant en évidence comment ces deux modes de relations se renforcent mutuellement.

Les ONG internationales au chevet de l'OUA/UA

24 Les relations d'interdépendance sont des interactions spécifiques «mutuellement coûteuses à interrompre ou à rompre $"^{32}$. Dans le domaine des droits humains, les organisations intergouvernementales et les ONG entretiennent des relations qui relèvent souvent de cette logique : même si celles-ci sont asymétriques, ces acteurs sont 
dans un rapport de dépendance mutuelle qui repose sur une contrainte partagée profitable en termes relatifs. Les ONG troquent ainsi leurs capacités d'expertise contre une entrée dans la sphère intergouvernementale et une plus grande influence sur la politique internationale; les organisations intergouvernementales bénéficient d'une ouverture sur la société civile, souvent fragile et incertaine, mais qui garde ses vertus légitimantes.

Si l'on trouve aujourd'hui de nombreuses études sur les relations entre les organisations intergouvernementales et les ONG, l'organisation politique africaine reste un terrain d'études en friche. L'OUA/UA a d'autant plus besoin des ONG qu'elle souffre d'un manque considérable de moyens. Mais au moment de la création de la Cour, l'OUA reste réticente à la participation des ONG à ses activités. Il n'y a à cela rien d'étonnant : une organisation créée pour défendre la souveraineté des Etats, et qui a été pendant de longues années un "syndicat de chefs d'Etat", a toutes les raisons de se méfier de ces nouveaux acteurs. Pour limiter le rôle des ONG sans s'opposer ouvertement à leur participation, l'OUA a mis au point un statut d'observateur qui restreint de facto leur accès ${ }^{33}$. Avant le passage à l'Union africaine, sur les cinquantequatre organisations bénéficiant d'un statut d'observateur, moins de dix avaient pour activité principale la défense des droits de l'Homme. Deux ONG internationales ont cependant obtenu des dérogations: la Commission Internationales des Juristes et l'African Society for International and Comparative Law. Ces organisations ont réussi à franchir toutes les étapes malgré les règles strictes de l'OUA grâce « à la personnalité de leurs dirigeants et aux gages de modération qu'ils acceptent de donner $»^{34}$. Pour les acteurs non étatiques qui participent à la création de la Cour, le passage par les canaux officiels de l'action des ONG est insuffisant. Celles-ci ont cependant d'autres cordes à leur arc. Nous verrons comment elles ont réussi à établir un véritable partenariat avec la Commission africaine des droits de l'Homme et des peuples avant d'étudier les relations de coopération qu'elles entretiennent avec le Secrétariat Général de l'OUA (aujourd'hui la Commission de l'UA).

Comme le souligne Kathryn Sikkink ${ }^{35}$, le processus normatif est circulaire : les acteurs non étatiques participent à la création de normes qui viennent en retour renforcer leur pouvoir et leur légitimité. La Commission africaine des droits de l'Homme est ainsi à la fois le fruit des efforts des ONG et un instrument pour les défenseurs des droits humains. Parce qu'elles sont les auteurs de la quasi-totalité des plaintes déposées, les ONG sont des éléments clés du mécanisme ${ }^{36}$. Lors de l'examen des rapports périodiques des Etats, ce sont les ONG qui préparent les rapports alternatifs qui permettent aux Commissaires d'engager le débat avec les représentants des Etats. Les ONG jouent également un rôle crucial dans la préparation des missions d'investigation sur le terrain ${ }^{37}$. Elles assurent enfin la publicisation et la médiatisation des résolutions prises par la Commission - un élément fondamental étant donné que « l'effet de honte » est la première sanction du gouvernement accusé de violer les droits humains.

En raison des difficultés financières de l'OUA/UA, les ressources allouées à la Commission des droits de l'Homme sont très insuffisantes. Cette situation précaire incite ses membres à accepter l'aide proposée par les ONG. Certains juristes qui travaillent auprès du Secrétariat permanent de la Commission sont ainsi recrutés et payés par des ONG et des fondations internationales. Il n'y a dès lors rien de nouveau dans le processus de la création de la Cour africaine : les ONG, et en particulier la CIJ, ont l'habitude de travailler derrière la scène. Alors que la résolution adoptée en 1994 
par l'Assemblée des chefs d'Etat et de gouvernement de l'OUA demande à la Commission africaine de se charger de l'élaboration d'une première version du Protocole, c'est la $\mathrm{CIJ}$ qui recrute les experts et orchestre le processus. Officiellement, la Commission est responsable de la création de la Cour, mais celle-ci n'aurait jamais vu le jour si la CIJ n'avait pas donné l'impulsion initiale et soutenu la Commission grâce à ses moyens financiers et ses capacités d'expertise.

28 A l'instar de la Commission africaine des droits de l'Homme, le Secrétariat Général de l'OUA ${ }^{38}$ a multiplié les partenariats avec les ONG. Ce phénomène n'est pas spécifique à l'organisation politique africaine: les bureaucraties des organisations intergouvernementales ont besoin des ONG pour pallier leur manque de ressources et être plus autonomes face aux Etats. Les logiques d'interdépendance sont cependant plus marquées dans le contexte africain en raison du dénuement matériel et financier de l'OUA/UA. Un ancien conseiller juridique d'une grande ONG internationale de protection des droits de l'Homme parle ainsi de son expérience :

«Les capacités d'expertise des ONG sont immenses comparées à celles du Secrétariat Général. Et c'est gratuit ! Après la réunion des experts sur la création de la Cour au Cap [en 1995], j'ai préparé des notes explicatives. Simplement les notes d'un expert d'une ONG. Le Secrétariat Général les a reprises et publiées avec la couverture officielle des documents de l'OUA $»^{39}$.

Certaines ONG professionnalisées prennent ainsi des initiatives qui sont reprises par une organisation qui a tout intérêt à profiter du travail qu'elles fournissent. Celles-ci bénéficient en retour de l'accès à une organisation intergouvernementale qui, si l'on s'en tient aux textes officiels, reste réfractaire à la participation des ONG.

$\mathrm{Au}$ sein de l'OUA, la Commission Internationale des Juristes a traditionnellement eu une place à part. Depuis le Congrès des juristes de Lagos en 1961, elle est très impliquée en Afrique. La $\mathrm{CIJ}$ bénéficie en outre d'un grand capital de confiance auprès des chefs d'Etat africains en raison de son long engagement en Afrique, de sa spécialisation dans l'aide juridique et de ses prises de positions très mesurées. Un accord est d'ailleurs signé entre la CIJ et l'OUA à l'automne 1996 pour officialiser cette collaboration ${ }^{40}$. Si la coopération entre les organisations intergouvernementales et les ONG est monnaie courante, les relations entre la CIJ et l'OUA sont marquées par leur longévité et leur institutionnalisation. De 1963 à $2001^{41}$, des générations de leaders des mondes étatiques et non étatiques se succèdent, renouvelant toujours le partenariat. La CIJ se trouve dans une situation de quasi-monopole : aucune autre ONG ne dispose des mêmes privilèges au sein de l'OUA. Les logiques d'interdépendance et les partenariats officiels ne suffisent cependant pas à expliquer le rôle qu'ont pu jouer les membres de la CIJ dans la création de la Cour : une collaboration aussi établie que celle de l'OUA et de la CIJ donne toujours naissance à des réseaux de notables.

Les réseaux de notables : un espace d'imbrication des deux mondes

31 Les " réseaux de sociabilité ${ }^{42}$ sont l'un des vecteurs de la formation d'un capital social et culturel qui peut s'avérer indispensable à la réalisation de certains projets ${ }^{43}$. Les chances de succès des entrepreneurs de normes non étatiques dépendent en grande partie de leur capacité à activer des réseaux de notables. Les notables de la scène africaine qui ont joué un rôle dans la création de la Cour sont des juristes, des responsables d'ONG africaines professionnalisées ou d'ONG internationales, des diplomates et des hommes et femmes politiques. Ces acteurs ont en commun d'être proches des réseaux de pouvoir et d'en connaître les codes. Certains d'entre eux jouent un rôle de pivot entre le monde étatique et le monde non étatique. Passant de l'un à 
l'autre ou appartenant aux deux mondes simultanément, ils sont des relais informels mais essentiels pour les acteurs non étatiques. Le parcours d'Ahmed Motala est à cet égard significatif: en 1994, il participe, en tant que membre d'une ONG sud-africaine, Lawyers for Human Rights, au groupe des cinq experts dits "non gouvernementaux" recrutés par la $\mathrm{CIJ}$ pour rédiger le Protocole portant création de la Cour. Un an plus tard, il rejoint le secrétariat d'Amnesty International à Londres. Mais la même année, il est aussi nommé chef de la délégation sud-africaine à la réunion des experts gouvernementaux du Cap, franchissant ainsi la frontière poreuse censée séparer le monde des ONG et celui des Etats. Les fonctions et les allégeances multiples des membres de la Commission africaine des droits de l'Homme ont ensuite été une ressource importante pour la création de la Cour - bien qu'elles soient par ailleurs l'une des principales raisons du manque d'indépendance de cette institution ${ }^{44}$. Le Commissaire tunisien qui a participé au premier groupe d'experts "non gouvernementaux » est aussi ambassadeur. Il est fort probable qu'il ait contribué à l'adoption de la résolution de l'oUA lançant officiellement le processus de la création de la Cour lors du sommet de Tunis en juin $1994^{45}$.

On ne retrouve donc pas une diplomatie des ONG qui serait une machine de guerre armée contre les Etats, mais plutôt des discussions et des négociations entre notables dans les couloirs de la Commission africaine des droits de l'Homme à Banjul (Gambie) et de l'OUA à Addis-Abeba (Ethiopie). Les réseaux informels qui lient les différents protagonistes ont joué un rôle considérable au moment de l'élaboration de la Charte africaine puis de la création de la Cour. L'ingénierie institutionnelle n'est alors pas seulement une affaire de spécialistes de la production normative, elle est aussi une activité réservée à un cercle restreint de notables maittrisant les codes du milieu à conquérir : celui des bureaucraties internationales et des diplomaties d'Etat.

L'avènement d'une diplomatie privée

33 Ayant pris acte de la porosité de la frontière entre le monde des Etats et le monde " multicentré », il nous reste à savoir si les membres des ONG ne sont pas en train de jouer, sur la scène africaine comme sur la scène internationale, le rôle qui était autrefois réservé aux diplomates ${ }^{46}$. Les acteurs non étatiques se professionnalisent et saisissent les opportunités de s'engager dans des activités diplomatiques aux côtés des Etats $^{47}$. Les diplomates « officieux » ou "privés », ou bien encore les "paradiplomates » sont de plus en plus nombreux. Les diplomaties d'Etat ne sont cependant pas pour autant tombées en désuétude. On assiste plutôt à un rapprochement croissant des activités des acteurs étatiques et non étatiques. Dans l'histoire de la création de la Cour africaine, nombreux sont les partenariats stratégiques entre acteurs étatiques et non étatiques. Les paradiplomates - la CIJ et les experts qu'elle recrute - ont tout d'abord organisé et encadré les négociations du Protocole ${ }^{48}$. Trois séries de réunions sont organisées au Cap en septembre 1995, à Nouakchott en avril 1997 et à Addis-Abeba en décembre 1997. Les réunions des experts non gouvernementaux précèdent à chaque fois celles des experts gouvernementaux. Les réunions des experts non gouvernementaux visent à discuter du Protocole et à mettre au point les stratégies des experts dits « indépendants » recrutés par la CIJ. Seuls les experts sélectionnés par la CIJ peuvent participer à ces réunions qui ne ressemblent en rien à des forums ouverts de débat. Quant aux réunions des experts gouvernementaux, elles sont l'espace de négociation du Protocole par les représentants des Etats membres de l'OUA. La fonction et l'identité des participants à ces deux types de réunions sont donc censées être très différentes. Le parallélisme est cependant frappant : elles se tiennent dans la même ville - et dans la 
même salle - à quelques jours d'intervalle. C'est le même discours du ministre de la Justice sud-africain, Dullah Omar, qui ouvre les deux réunions du Cap! De nombreuses personnalités participent aux deux types de réunions : les représentants de l'OUA, les Commissaires, mais aussi certains membres d'ONG. Il faut en outre noter que toutes les réunions - même celles des experts gouvernementaux - sont organisées avec la collaboration de la $\mathrm{CIJ}$ qui joue ainsi le rôle d'une diplomatie privée.

Le manque de ressources financières de l'OUA est une première explication de la position dominante de la $\mathrm{CIJ}$ dans le processus. Une ancienne conseillère juridique de la Commission africaine des droits de l'Homme nous fait part de ce qu'elle a pu observer :

« La CIJ avait peut-être plus d'argent que l'OUA. Quand il s'agissait d'organiser une réunion, la $\mathrm{CIJ}$ pouvait dire : 'nous sommes en relation avec des bailleurs de fonds, nous pouvons partiellement la financer à votre place.' L'OUA manque de moyens financiers et est très désorganisée. (...) Mais quand les ONG s'impliquent, il devient possible de les amener à faire un bon travail $»^{49}$.

D'une manière générale, la participation des acteurs non étatiques aux négociations internationales a été possible à partir du moment où l'activité diplomatique est passée de la recherche d'un arrangement entre Etats dans un jeu à somme nulle à la création d'une norme complexe. Dans le domaine des droits humains, les négociations n'impliquent pas seulement des marchandages entre Etats mais aussi la création de nouveaux savoirs, l'énonciation de principes généraux et la formation de ceux qui ne maitrisent pas les dernières technologies juridiques. La marge de manœuvre des experts dits «indépendants » varie cependant beaucoup d'une négociation à l'autre. Dans le cas de la Cour africaine, non seulement l'OUA attribue à une ONG internationale le statut de co-organisateur des réunions des délégués gouvernementaux, mais elle accepte aussi que les négociations se basent sur le texte produit par des acteurs non étatiques ${ }^{50}$. Les réunions des experts non gouvernementaux préparent en effet une version du Protocole qui est ensuite transmise aux experts gouvernementaux.

Le statut de co-organisateur de la CIJ lui a permis d'«inviter» des experts « indépendants » aux négociations. Ils assistent en tant qu'observateurs aux réunions des experts gouvernementaux et n'ont pas le droit de prendre la parole. Leur rôle n'est cependant pas celui d'un figurant: ils s'entretiennent avec les experts gouvernementaux et les membres de la Commission des droits de l'Homme avant les réunions et durant les pauses. La meilleure stratégie consiste cependant à user de contacts personnels, comme nous l'explique l'un de ces experts « indépendants »:

«En décembre 1997 [à la troisième réunion des experts gouvernementaux], j'étais observateur, je n'étais pas autorisé à parler. Néanmoins, dans ce genre de réunions, les observateurs peuvent avoir des contacts avec certaines délégations et faire passer des messages par ce canal. Vous préparez des amendements et vous les donnez à un expert gouvernemental qui va présenter une motion ou proposer la révision de tel paragraphe ou de telle phrase dans le sens que vous souhaitez $»^{51}$.

Pour cet "observateur ", ce sont avant tout les capacités d'expertise des experts non gouvernementaux qui sont recherchées par les représentants des Etats :

«Les délégués gouvernementaux manquent souvent d'expertise. Tout le monde n'a pas accès au fonctionnement d'une Cour comme la Cour européenne par exemple. Dans le milieu des ONG et des organisations internationales, on est plus familier de ce genre de procédures. (...) Et les délégués des gouvernements ne sont évidemment pas opposés à l'idée de recevoir des contributions des observateurs! »

Il s'agit donc d'experts « techniques » qui, en raison de leurs capacités d'expertise et de leur appartenance à des réseaux de sociabilité, sont capables de peser sur le 
déroulement des négociations. Les experts, par leurs conseils aux délégués gouvernementaux, participent à la production de normes même si leur contribution est moins nette à l'étape des négociations qu'à celle de la production de la première version du texte. Ce phénomène n'a rien d'extraordinaire : les ONG possèdent souvent une plus grande expertise scientifique et technique que les gouvernements ou le secrétariat des Nations Unies ${ }^{52}$. Sans besoins mutuels et sans dialogue, la contribution des acteurs non étatiques à l'écriture du droit serait impossible. Mais les diplomaties africaines, en raison de leur manque de ressources, sont susceptibles d'être plus ouvertes aux conseils des experts non gouvernementaux. Lors des négociations du Protocole de Ouagadougou, comme lors de celles du Statut de la Cour Pénale Internationale ${ }^{53}$, les Etats les moins bien dotés sont amenés à pallier leurs faiblesses en ayant recours à l'aide juridique fournie par les ONG. Les Etats africains ont en outre peu de moyens de pression sur les ONG internationales qui sont nées sur d'autres continents et bénéficient parfois du soutien des Etats les plus puissants et des financiers internationaux, les agents d'une nouvelle "supra-diplomatie», pour reprendre la formule d'Ariel Colonomos ${ }^{54}$.

L'exigence de faire « bonne figure » sur la scène internationale

39 Les Etats africains ont joué le jeu de la construction de la Cour africaine. L'attitude des gouvernements à l'égard de la Commission africaine des droits de l'Homme ne laissait pourtant pas présager d'un tel dénouement. Comme nous l'avons vu, les capacités des acteurs non étatiques à collaborer avec la Commission africaine des droits de l'Homme et le Secrétariat Général de l'OUA, ainsi que l'activation des réseaux de notables ont été des éléments déterminants dans la réalisation du projet. Ils ne constituent cependant qu'une partie de l'explication. Les Etats ne sont en effet jamais totalement contraints de se laisser guider par les ONG. Certains gouvernements célèbres pour leur non-respect des droits humains se sont engagés dans le processus de la création de la Cour africaine parce qu'ils n'étaient pas prêts à payer le coût, en termes d'image, d'une opposition directe à la future juridiction. Quant à ceux qui jouent la carte de la promotion des droits humains (l'Afrique du Sud par exemple), ils y ont trouvé une nouvelle occasion d'affirmer leur attachement à cette norme. Sur la scène internationale, comme dans la vie quotidienne, il importe de faire «bonne figure $~^{55}$.

Du côté des Etats : manque de ressources, faible intérêt et mauvaise volonté

40 Avant de célébrer un engagement inattendu des gouvernements, il convient de rappeler que l'option juridictionnelle ne représente pas, de leur point de vue, un enjeu majeur. La création de la Cour n'est pas perçue comme une menace directe et immédiate par les gouvernements qui violent les droits humains. Mais parce qu'elle peut devenir une nouvelle tribune pour les défenseurs des droits de l'Homme et une nouvelle source "d'embarras" pour les Etats, ceux qui ont le plus à craindre de la création d'une juridiction veulent s'assurer de ne pas avoir affaire à une institution trop puissante ou trop indépendante. Ils ont alors recours à des stratégies plus discrètes que l'opposition frontale pour affaiblir le Protocole.

41 Si aucun gouvernement ne cherche à bloquer les négociations, le processus de la création de la Cour est sans cesse ralenti par la très faible participation des acteurs étatiques. Nombreux sont les Etats qui ne sont pas représentés aux réunions des experts gouvernementaux du Cap et de Nouakchott. Seuls quelques Etats prennent la peine d'envoyer leurs commentaires sur le Protocole au Secrétariat général de l'OUA, comme l'exige la procédure ${ }^{56}$. Pour ces raisons, les échéances doivent être maintes fois 
repoussées. Une première hypothèse peut être avancée : les Etats africains, qui sont très sollicités pour l'élaboration de standards juridiques aux niveaux régional et international, ne disposent pas toujours d'experts compétents. C'est pourquoi la troisième réunion des experts gouvernementaux élargie aux diplomates, qui se tient à Addis-Abeba, au siège de l'OUA - où la quasi-totalité des Etats membres ont une représentation permanente - a accueilli beaucoup plus de participants. Le manque de moyens humains et financiers était déjà l'une des explications de la faible participation des Etats africains aux premières étapes de l'élaboration du Statut de la CPI ${ }^{57}$. Les questions d'organisation ne doivent donc pas être sous-estimées, surtout quand il s'agit de l'OUA et de l'UA. Une explication alternative peut être que la création de la Cour n'est pas un enjeu mobilisateur : elle n'est en effet pas une priorité des Etats africains mais une initiative des ONG relayée par l'OUA. La faible participation des Etats peut enfin être interprétée comme le résultat d'une stratégie délibérée de ralentir le processus. Les trois hypothèses sont évidemment compatibles: certains Etats ont pu choisir de ne pas participer au processus par manque d'intérêt et/ou de ressources, d'autres par hostilité au projet. Pour les gouvernements réticents à la création de la Cour, l'absentéisme n'est de toute façon pas la meilleure des stratégies: elle est difficilement justifiable à long terme et surtout risquée puisqu'elle laisse la négociation entre les mains des partisans de la Cour. La future institution ne constituant pas une grande menace, il est beaucoup plus judicieux de ne pas se compromettre dans une entreprise de blocage du processus et de ruser pour affaiblir le texte.

Les ruses des Etats hostiles à la Cour pour affaiblir le Protocole

Le propre du droit international est de conduire les Etats à respecter les normes qu'ils ont eux-mêmes produites. Mais pour alléger la contrainte que représentent les institutions de protection des droits humains, ils peuvent tenter de façonner la production normative au moment des négociations. Affaiblir un texte de droit est en effet moins coûteux en termes d'image que de s'y opposer ouvertement ou de refuser d'y adhérer. C'est la stratégie choisie pas les Etats les plus réticents au projet de la Cour. D'après l'un des experts non gouvernementaux qui a participé aux négociations, «les Etats ont accepté le projet mais ils se sont arrangés pour le modifier de façon très significative $\aleph^{58}$.

Certaines modifications du texte initial témoignent des efforts entrepris par les représentants des Etats hostiles à la Cour pour affaiblir la future institution. La question de la saisine de la Cour a été la plus controversée. Dans la version du Protocole rédigée par les experts non gouvernementaux, les ONG et les individus peuvent saisir la Cour, "dans des circonstances exceptionnelles" sans passer par le filtre de la Commission africaine des droits de l'Homme ${ }^{59}$. Certaines délégations, celles du Nigeria et de l'Egypte par exemple, se sont opposées à cet article qui aurait donné des pouvoirs considérables à la nouvelle institution. Les experts réunis à Nouakchott se sont finalement accordés sur une clause facultative que les Etats peuvent ratifier s'ils souhaitent autoriser la saisine de la Cour par les ONG et les individus ${ }^{60}$. De l'avis d'un membre de la Commission africaine : "le texte ne serait jamais passé sans cette clause de sauvegarde $»^{61}$.

Le Protocole, sensiblement affaibli, a finalement été adopté puis officiellement entériné par les chefs d'Etat et de gouvernement lors du sommet de Ouagadougou le 9 juin 1998. Vingt-quatre Etats signent alors le Protocole qui n'entre en vigueur que le 25 janvier 2004. 
Les effets vertueux inattendus des changements de façade? entrepreneurs de normes, sur les effets possibles de la création de cette nouvelle juridiction pour la protection des droits humains en Afrique. La Cour ne se substitue pas à la Commission africaine des droits de l'Homme et des peuples : les deux institutions ont vocation à se compléter. La nouvelle juridiction peut être saisie par la Commission, les Etats parties et les organisations intergouvernementales africaines. Les individus et les ONG peuvent, en cas d'épuisement des voix de recours internes, introduire des requêtes directement devant la Cour si et seulement si l'Etat concerné a ratifié l'article $34.6 \mathrm{du}$ Protocole. A ce jour, seul le Burkina Faso autorise la saisine directe des individus et des ONG. La Commission jouera donc dans la plupart des cas un rôle d'interface entre la Cour d'une part et les individus et les ONG d'autre part. On peut alors redouter que les faiblesses de la Commission entravent le fonctionnement de la future juridiction. Le pouvoir et l'indépendance de la Cour dépendront également du processus de l'élection des onze juges ${ }^{62}$, du règlement intérieur, du budget et des moyens de fonctionnement, de la volonté des Etats de coopérer avec elle, mais aussi de la réappropriation de ce nouvel instrument par les militants africains.

En juillet 2004, les chefs d'Etat et de gouvernement de l'Union africaine ont décidé de fusionner la Cour africaine des droits de l'Homme et des peuples et la Cour de justice de l'UA. Cette décision a déjà retardée la mise en place effective de la Cour des droits de l'Homme, mais elle pourrait aussi remettre en cause ses spécificités en matière de protection des droits humains. La juridictionnalisation du mécanisme africain ne saurait donc être un élément suffisant pour une protection efficace des droits de l'Homme.

47 L'entrée en vigueur du Protocole portant création de la Cour est cependant le signe d'une évolution significative du climat politique sur le continent. Cet événement s'inscrit dans le contexte de la production de nouveaux discours et de la mise en place de nouvelles institutions au sein de l'organisation politique africaine. Alors que la Charte de l'OUA insistait sur la libération de l'Afrique du colonialisme et sur la souveraineté des Etats, l'Acte Constitutif de l'Union africaine place la défense des droits humains et le respect de la démocratie au cœur du projet régional ${ }^{63}$. Les institutions censées protéger les droits humains se multiplient sur le continent : un Comité africain d'experts sur les droits et le bien-être de l'enfant s'est réuni pour la première fois en avril $2002^{64}$ tandis que le Protocole additionnel à la Charte africaine relatif aux droits de la femme en Afrique est entré en vigueur en novembre 2005.

48

Si ces récents changements de discours et ces innovations institutionnelles relevaient de simples exercices de relations publiques, devrait-on pour autant les réduire à des phénomènes sans importance, les condamner à ne jamais avoir d'effets ? L'adoption du discours des droits humains est devenue un mode courant de légitimation politique sur la scène internationale. Le poids des normes, qui se manifeste dans la volonté d'appartenir à une communauté normative et dans la crainte des sanctions réservées aux «déviants $»^{65}$ de la scène internationale, incite les Etats et les organisations intergouvernementales à accepter des changements de façade. L'histoire nous a cependant enseigné à maintes reprises que des institutions vulnérables au moment de leur création peuvent se développer, s'autonomiser et prendre une importance inattendue. En ratifiant la Charte africaine et ses Protocoles additionnels, les Etats reconnaissent les droits humains et s'engagent dans un dialogue avec les acteurs 
nationaux et transnationaux de défense des droits de l'Homme. Les Etats sont alors pris dans un "filet normatif " dont les mailles se resserrent, selon les termes d'Antonio Cassese $^{66}$. Les engagements des Etats deviennent de nouveaux leviers pour les entrepreneurs de normes qui peuvent souligner l'écart entre les discours et les pratiques et obliger les dirigeants des Etats à se justifier et à s'engager ainsi encore un peu plus dans la rhétorique des droits humains. Les Etats sont pris dans l'engrenage des rhétoriques et des procédures. L'instrumentalisation totale n'existe pas: si la supercherie est trop visible, ils détruisent leur propre instrument de légitimation.

\section{NOTES}

1.. Colonomos A., « Non-state Actors as Moral Entrepreneurs », in Josselin D., Wallace W., Non-state Actors in World Politics, New York, Palgrave, 2001, pp. 76-89.

2.. Dorandeu R., « Les pèlerins constitutionnels. Eléments pour une sociologie des influences juridiques », in Mény Y. (dir.), Les Politiques du mimétisme institutionnel, la greffe et le rejet, Paris, L'Harmattan, 1993, pp. 83-112.

3.. Keck M., Sikkink K., Activists Beyond Borders, Advocacy Networks in International Politics, Ithaca, Cornell University Press, 1998.

4.. A ce jour, vingt-deux Etats ont ratifié le Protocole. Voir la liste sur le site Internet de l'Union africaine : www.africa-union.org

5.. L'Union africaine a remplacé l'Organisation de l'unité africaine (OUA). L'acte constitutif de l'UA a été adopté en 2000 lors du Sommet de Lomé au Togo. L'Union africaine a pris officiellement la succession de l'OUA le 9 juillet 2002 lors du sommet de Durban (Afrique du Sud). Dans cet article, nous parlerons cependant de l'OUA car le Protocole portant création de la Cour a été rédigé, négocié et adopté avant le passage à l'UA.

6.. Le terme de « monde multicentré » a été forgé par James Rosenau. Rosenau J.N., Turbulence in World Politics. A Theory of Change and Continuity, Princeton, Princeton University Press, 1990.

7.. Melissen J. (dir.), Innovation in Diplomatic Practice, Londres, MacMillan Press LTD, 1999.

8.. Gabou A., « Principes et mécanismes de protection des droits de l'Homme et des peuples : la Charte de Banjul », Revue Congolaise de droit, n¹0, juillet-décembre 1991, pp. 98-122.

9.. Badie B., La Diplomatie des droits de l'Homme, Paris, Fayard, 2002, p. 119.

10.. Mény Y., « La greffe et le rejet. Les politiques du mimétisme institutionnel », in Mény Y. (dir.), Les Politiques du mimétisme institutionnel, la greffe et le rejet, Paris, L'Harmattan, 1993, pp. 14-15.

11.. Mbaye K., Les Droits de l'Homme en Afrique, Paris, Editions A. Pedone, 1992.

12.. Welch C.E, Protecting Human Rights in Africa, Role and Strategies of Non-Governmental Organizations, Philadelphie, University of Pennsylvania Press, 1995, p. 177.

13.. La Charte africaine a été ratifiée par tous les Etats membres de l'OUA/UA, soit cinquante-trois Etats. Le Maroc, qui s'est retiré de l'OUA en 1984 pour protester contre la reconnaissance de la République Arabe Sahraouie Démocratique, n'est pas partie au traité. 
14.. Sur les relations entre la Commission africaine des droits de l'Homme et la Cour, voir infra.

15.. Mény Y., « La greffe et le rejet. Les politiques du mimétisme institutionnel », op. cit.

16. Voir les articles 48 et 55 de la Charte.

17.. Mubilia M., « La Cour africaine des droits de l'Homme et des peuples : mimétisme institutionnel ou avancée judiciaire ? ", Revue générale de droit international public, vol. 102, n³, 1998, pp. 765-780.

18.. Sur le rôle de la Commission africaine et les limites de ses actions voir Evans M.D, Murray R. (dir.), The African Charter on Human and People's Rights : the System in Practice, 1986-2000, Cambridge, Cambridge University Press, 2002 ; Hansungule M., « The African Charter on Human and Peoples' Rights : a critical review ", African Yearbook of International Law, vol. 8., 2000, pp. 265-331 ; Olinga A.D., « L'effectivité de la Charte Africaine des droits de l'Homme et des peuples ", Afrique 2000, n²7-28, avril-octobre 1997, pp. 171-185; Welch C.E, « The Organisation of the African Unity and the Promotion of Human Rights ", The Journal of Modern African Studies, vol. 29, n4, décembre 1991, pp. 535-555.

19.. Selon la définition de nombreux auteurs menant des recherches sur les normes en relations internationales : Alexander Wendt, Peter Katzenstein, Audie Klotz, Margaret Keck, Kathryn Skikkink, Thomas Risse, etc. Voir, par exemple, Klotz A., Norms in International Relations, The Struggle Against Apartheid, Ithaca and London, Cornell University Press, 1995.

20.. Voir à titre d'exemple la « déclaration sur la situation politique et socioéconomique de l'Afrique et sur les changements fondamentaux qui affectent le monde » adoptée par l'Assemblée des chefs d'Etat et de gouvernement lors de sa $26^{\mathrm{e}}$ session ordinaire à Addis-Abeba en 1990.

21.. Après l'arrêt de la Cour Internationale de Justice dans l'affaire de Sud-Ouest africain en 1966, les Etats africains se méfient des juridictions internationales. La situation change dès les années 1980 avec la saisine de la Cour Internationale de Justice pour les différends territoriaux entre le Burkina Faso et la Mali (1986) et entre la Libye et la Tchad (1994). Burgorgue-Larsen L., « Le fait régional dans la juridictionnalisation du droit international ", in Société Française pour le Droit International, La juridictionnalisation du droit international, Paris, Pédone, 2003, (Colloque de Lille), pp. 203-264.

22.. Maqungo S., « The African contribution towards the establishment of an International Criminal Court », African Yearbook of International Law, vol. 8, 2000, pp. 333-350.

23.. Il s'agit de la Conférence mondiale des Nations Unies sur les droits de l'Homme en 1993.

24.. Entretien réalisé le 26 juin 2003.

25.. Dezalay Y., Garth B., « Droits de l'homme et philanthropie hégémonique », Actes de la recherche en sciences sociales, $\mathrm{n}^{\circ} 121-122$, mars 1998, pp. 23-41.

26. Entretien avec un haut fonctionnaire du Haut Commissariat aux droits de l'Homme de l'ONU, réalisé le 26 juin 2003.

27.. Commission Internationale des Juristes, ICJ Workshops on NGO Participation in the African Commission on Human and Peoples'Rights, 1991 to 1996. A Critical Evaluation, Genève, 1996.

28.. Ces experts sont dits « non gouvernementaux », bien que l'un d'entre eux soit diplomate... Voir infra. 
29.. Entretien réalisé le 21 mars 2003.

30.. "The ICJ is not and was never a spontaneous citizens' movement organized to challenge state power. (...) The ICJ more closely resembles quasi-nongovernmental organizations that use public funds to provide a public service ». Tolley H.B., The International Commission of Jurists, Global Advocates for Human Rights, Philadelphie, University of Pennsylvania Press, 1994, p. 278.

31.. Rosenau J.N., Turbulence in World Politics, op.cit.

32.. Risse-Kappen T., « Bringing Transnational Relations Back In : Introduction » in Risse-Kappen T. (dir.), Bringing Transnational Relations Back In. Non-state Actors, Domestic Structures and International Institutions, Cambridge, Cambridge University Press, 1995, p. 8.

33. Il faut que cinq Etats membres soutiennent l'ONG et qu'aucun autre ne s'oppose à son accession au statut d'observateur. L'organisation doit être " panafricaine ", c'est à dire avoir son siège dans un pays du continent, être composée en majorité d'Africains, mais aussi tirer la majorité de ses ressources financières du continent. Difficile d'imaginer des conditions plus restrictives! Les critères à remplir pour obtenir le statut d'observateur auprès de l'OUA, avec les amendements de la $29^{\circ}$ session ordinaire de l'Assemblée des chefs d'Etat et de Gouvernements, sont reproduits dans Garling M., Odinkalu C.A., Building Bridges for Rights InterAfrican Initiatives in the Field of Human Rights, Interights, Londres, 2001, pp. 143-146. Les critères d'octroi du statut d'observateur auprès de l'UA ont été modifiés en juillet 2005. Le nouveau texte est disponible sur le site Internet de l'organisation : www.africa-union.org.

34. Entretien avec un membre de la Commission africaine des droits de l'Homme et des peuples. Le 4 juillet 2003.

35.. Sikkink K., « Transnational advocacy networks and the social construction of legal rules ", in Dezalay Y., Garth B., Global Prescriptions, The Production, Exportation, and Importation of a New Legal Orthodoxy, The University of Michigan Press, 2002, p. 48. 36.. Les conditions à remplir pour obtenir le statut d'observateur auprès de la Commission africaine des droits de l'Homme sont beaucoup moins restrictives que celles requises pour obtenir un statut équivalent auprès de l'OUA et de l'UA. La résolution de la Commission sur les nouveaux critères à remplir pour obtenir le statut d'observateur, adoptée lors de sa $25^{\mathrm{e}}$ session ordinaire en mai 1999, est reproduite dans Garling M., Odinkalu C.A., Building Bridges for Rights, op. cit., pp. 147- 150.

37.. Motala A., « Non-governmental organisations in the African system », Evans M.D., Murray R. (dir.), The African Charter on Human and People's Rights, op. cit., pp. 262-263.

38. Cette remarque concerne également la Commission de l'UA qui a remplacé le Secrétariat Général de l'OUA.

39.. Entretien réalisé le 28 mars 2003. Un responsable d'une autre ONG dit avoir été plusieurs fois témoin de la reprise des rapports des experts non gouvernementaux par le Secrétariat Général de l'OUA.

40.. Bovay N., Prouvez N., "The role of the International Commission of Jurists », in Welch C.E. (dir.), NGOs and Human Rights, Promise and Performance, Philadelphie, University of Pennsylvania Press, 2001., p. 132.

41.. Le bureau Afrique de la CIJ disparaît de façon assez inattendue en 2001.

42.. Forsé M., «Les réseaux de sociabilité, un état des lieux ", L'Année sociologique, $\mathrm{n}^{\circ} 41$, 1991, pp. 245-264.

43.. Dezalay Y., Garth B., " Droits de l'Homme et philanthropie hégémonique », op. cit. 44.. Les Commissaires sont nommés par leurs Etats et élus par l'Assemblée des chefs d'Etat et de gouvernement (aujourd'hui la Conférence des chefs d'Etat et de 
gouvernement). Nombreux sont ceux qui cumulent les fonctions de Commissaire et d'ambassadeur ou ministre.

45.. La résolution AHG/203 (XXX) invite le Secrétaire Général de l'OUA à « convoquer une réunion d'experts gouvernementaux pour examiner, avec la Commission africaine des droits de l'Homme et des peuples, les moyens de renforcer l'efficacité de la Commission et envisager en particulier la création d'une Cour africaine des droits de l'Homme et des peuples ». 46.. Devin G., "Les diplomaties de la politique étrangère ", in Charillon F., Politique Etrangère, Nouveaux regards, Paris, Presses de Sciences Po, 2002, pp. 215-242.

47.. Cohen S. (dir.), Les Diplomates. Négocier dans un monde chaotique, Paris, Autrement, 2002.

48. Tous les traités adoptés par l'OUA sont soumis à la même procédure. Une première résolution de l'Assemblée des chefs d'Etat et de gouvernement lance officiellement le processus de l'élaboration du texte. Des experts gouvernementaux discutent ensuite du texte qui est soumis aux Etats membres pour commentaires. Le texte modifié est alors approuvé par le Conseil des ministres des affaires étrangères avant l'adoption finale par l'Assemblée des chefs d'Etat et de gouvernement.

49.. " The ICJ might have more money than the OAU. So if it comes to convening a meeting, the ICJ might say "you know, we have connection with donors, we can partially fund it for you". The $O A U$ is not very well-funded and very disorganized (...). So, if you involve NGOs, you can get them to do a lot of work ». Notre traduction. Entretien réalisé le 15 mai 2003.

50.. Il est intéressant de noter que Jean Mouton-Brady écrivait en 1986 :

"L'investissement des organisations intergouvernementales par les ONG sera complet lorsque celles-ci, ne se contentant plus de coopérer de l'extérieur à l'action des institutions internationales, participeront à leurs travaux de l'intérieur, à côté des délégations gouvernementales ». C'est bien ce phénomène que l'on observe actuellement! MoutonBrady J., « La contribution des organisations non gouvernementales à la formation et à l'application des normes internationales, à travers les instances gouvernementales et intergouvernementales ", in Bettati M., Dupuy P-M.

(dir.), Les ONG et le Droit International, Paris, Economica, 1986, p. 56.

51.. Entretien avec un haut fonctionnaire du Haut Commissariat aux droits de l'Homme de l'ONU, réalisé le 26 juin 2003.

52.. Spector G.I, Sjöstedt G., Zartman W. (dir.), Negotiating International Regimes : Lessons learned from the United Nations Conference on Environment and Development (UNCED), Londres, Graham and Trotman, 1994.

53.. Törnquist-Chesnier M., Expertise et éthique dans la fabrication du droit international public : la contribution des organisations non gouvernementales : trois cas d'étude, thèse de doctorat de science politique, sous la direction de Guillaume Devin, Institut d'Etudes Politiques de Paris, 2004, 512 p.

54.. Colonomos A., « Non-state Actors as Moral Entrepreneurs: A Transnational Perspective on Ethics Networks », op. cit, p. 77.

55.. Goffman E., Les Rites d'interaction, Paris, Editions de Minuit, 1974.

56.. Seuls vingt-trois Etats sont représentés à la réunion des experts gouvernementaux du Cap. Le Protocole issu des discussions des experts est ensuite transmis aux Etats afin qu'ils soumettent leurs commentaires. Mais seuls quatre Etats daignent envoyer leurs commentaires avant la tenue de la $64^{\mathrm{e}}$ session du Conseil des ministres de l'OUA en juillet 1996. En raison de la faible participation des Etats, le Conseil des Ministres décide de repousser l'examen du Protocole à la session suivante, six mois plus tard. En février 1997, seuls dix Etats ont soumis leurs commentaires sur le Protocole. Le Conseil des 
ministres décide alors de la tenue d'une seconde réunion d'experts à Nouakchott. Mais seuls dix-neuf Etats membres envoient une délégation dans la capitale mauritanienne. Le Conseil des ministres, réuni au Zimbabwe en mai 1997, décide une nouvelle fois de repousser l'examen du Protocole et demande l'organisation d'une troisième réunion d'experts gouvernementaux. Cette dernière réunion qui se tient à Addis-Abeba accueille, sur décision de l'OUA, des diplomates qui n'ont pas un statut d'expert. La participation des diplomates annonce le passage à une négociation moins technique et plus politique. Cent treize délégués de quarante-cinq pays participent alors à la réunion qui adopte le Protocole à l'unanimité.

57.. De nombreux Etats du continent ont en effet commencé à participer aux diverses commissions préparatoires après la mise en place d'un fond d'aide aux Etats les plus pauvres en décembre 1996. Ces Etats ont formé un groupe opposé au contrôle de la CPI par le Conseil de Sécurité de l'ONU qui a pu peser sur l'issue des négociations. Maqungo S., « The African contribution towards the establishment of an International Criminal Court », op. cit, p. 347.

58.. Entretien réalisé le 28 mars 2003.

59.. Le Protocole rédigé par les experts non gouvernementaux était très ambitieux. En Europe, il a fallu attendre l'entrée en vigueur du Protocole 11 en 1998 et la mise en place de la Cour unique pour que les individus et les ONG soient autorisés à saisir directement la Cour.

60.. Cette déclaration peut être faite à tout moment à partir de la ratification.

61.. Entretien réalisé le 4 juillet 2003.

62.. Les onze juges de la cour sont élus par les Etats membres de l'Union africaine pour un mandat de six ans, renouvelable une seule fois (articles 11, 12 et 15 du Protocole) 63.. Dans le préambule de l'Acte constitutif de l'Union africaine, les chefs d'Etat et de gouvernement s'engagent à " promouvoir et à protéger les droits de l'Homme et des peuples, à consolider les institutions et la culture démocratiques, à promouvoir la bonne gouvernance et l'Etat de droit ".

64.. Le Protocole sur les droits et le bien-être de l'enfant adopté en juillet 1990 est entré en vigueur en novembre 1999. Lloyd A., «The first meeting of the African Committee of Experts on the Rights and Welfare of the Child ", African Human Rights Law Journal, vol. 2, n² 2, 2002, pp. 320-326.

65.. Becker H., Outsiders. Etudes de sociologie de la déviance, Paris, Editions A-M. Metailié, 1985.

66.. Cassese A., Violence et droit dans un monde divisé, Paris, PUF, 1990, p. 13.

\section{RÉSUMÉS}

L'étude du processus d'ingénierie institutionnelle de la Cour africaine des droits de l'Homme et des peuples révèle le rôle central joué par les ONG : elles ont donné l'impulsion initiale, orchestré la mobilisation, encadré les négociations et milité pour l'adoption du Protocole. Ce nouveau mode de production du droit doit cependant être analysé avec un regard critique afin de déceler les logiques de pouvoir très fortes au sein $\mathrm{du}$ « monde multicentré ». La professionnalisation des 
ONG et l'autonomisation du champ de la production des standards juridiques vont de pair avec la marginalisation des militants locaux. L'activité de production de normes est l'affaire des experts juridiques apolitiques qui entretiennent des relations de collaboration avec les notables des diplomaties d'Etat et des organisations intergouvernementales.

Studying the process of the institutional engineering of the African Court on Human and peoples' rights reveals the central role played by NGOs: they gave the initial impulse, orchestrated the mobilisation, supervised the negotiations and militated for the adoption of the Protocol. However, this new mode of producing law must be analysed with a critical view in order to detect the logics of power pervading the "multicentric world". The professionalisation of NGOs and the autonomisation of the field of production for legal rules go hand in hand with the marginalisation of local activists. The activity of norms production becomes a matter taken up by apolitical legal experts who maintain forms of collaboration in their relations to the notables of state diplomacies and intergovernmental organisations.

\section{INDEX}

Thèmes : Union africaine (Organisation de $l^{\prime}$ )

Mots-clés : cours et tribunaux, droits de l'Homme, juge, régionalisme et régionalisation

Index géographique : Afrique australe, Afrique de l'ouest, Afrique subsaharienne, Corne de

l'Afrique

\section{AUTEUR}

\section{MARIELLE DEBOS}

Marielle Debos est doctorante en science politique (relations internationales) à l'Institut d'Etudes Politiques de Paris. Allocataire de recherche et monitrice, elle enseigne les relations internationales à l'IEP de Paris. marielle.debos@sciences-po.org. 